\title{
Transatlantica
}

Revue d'études américaines. American Studies Journal

2 | 2014

Aesthetics of Theory in the Modern Era and Beyond / Photographie documentaire

Juliette Bourdin, Entre porte ouverte et « porte fermée » : la politique chinoise des États-Unis du XIX ${ }^{e}$ au XXI ${ }^{e}$ siècle

Paris, Presses Sorbonne nouvelle, 2013

\section{Nelly Mok}

\section{(2) OpenEdition}

Journals

Édition électronique

URL : https://journals.openedition.org/transatlantica/7205

DOI : $10.4000 /$ transatlantica.7205

ISSN : 1765-2766

Éditeur

Association française d'Etudes Américaines (AFEA)

Référence électronique

Nelly Mok, « Juliette Bourdin, Entre porte ouverte et «porte fermée » : la politique chinoise des États-Unis du XIXe au XXIe siècle », Transatlantica [En ligne], 2 | 2014, mis en ligne le 19 février 2015, consulté le 31 janvier 2023. URL : http://journals.openedition.org/transatlantica/7205 ; DOI : https://doi.org/ 10.4000/transatlantica.7205

Ce document a été généré automatiquement le 31 janvier 2023.

\section{cc) $(9)$}

Creative Commons - Attribution - Pas d'Utilisation Commerciale - Pas de Modification 4.0 International - CC BY-NC-ND 4.0

https://creativecommons.org/licenses/by-nc-nd/4.0/ 


\title{
Juliette Bourdin, Entre porte ouverte et " porte fermée " : la politique chinoise des États-Unis du XIX ${ }^{e}$ au XXI ${ }^{e}$ siècle
}

Paris, Presses Sorbonne nouvelle, 2013

\author{
Nelly Mok
}

\section{RÉFÉRENCE}

BOURDIN, Juliette, Entre porte ouverte et " porte fermée » : la politique chinoise des États-Unis du XIXe au XXI e siècle, Paris, Presses Sorbonne nouvelle, 2013, 272 pages, ISBN

978-2-87854-591-3, 22 euros.

\section{Résumé de l'ouvrage}

1 Dans cet ouvrage, Juliette Bourdin se propose de mettre en lumière les tendances caractéristiques des relations sino-américaines depuis leur émergence à l'occasion des toutes premières percées américaines dans le marché chinois dès la fin du XVIII siècle jusqu'à nos jours. Par le biais d'une approche chronologique, l'auteure entreprend d'identifier l'héritage historique des relations sino-américaines tel qu'il s'est constitué au fil des deux derniers siècles, afin de mieux appréhender les enjeux actuels et les prolongements potentiels de ces relations. De cette façon, elle entend aussi et surtout souligner la résurgence de lignes de conduite et de stratégies diplomatiques, économiques et militaires chez l'une et l'autre partie au cours de l'histoire du dialogue sino-américain, mettant en avant la « dimension cyclique d'ouverture et de fermeture " (20) des relations entre les deux pays, comme l'annonce le titre de l'ouvrage : à travers une perspective américaniste - néanmoins mise en regard avec un éclairage essentiel sur le contexte historique et socio-politique asiatique et chinois - l'auteure s'interroge sur les divers facteurs à l'origine de la nature ambivalente, souvent versatile, des 
échanges sino-américains. Sont alors évoquées, dans les sept parties qui structurent l'ouvrage, les raisons aussi bien conjoncturelles, inhérentes à l'évolution historique du contexte géopolitique international, que structurelles, intrinsèques à la culture et à l'idéologie sous-tendant les sociétés chinoise et américaine, de l'oscillation des deux pays entre coopération et hostilité, amitié et inimitié.

2 La première partie s'intéresse aux origines des relations sino-américaines, nées du premier voyage commercial du navire américain Empress of China sur les côtes chinoises en 1784, et identifie les fondements de la politique chinoise des États-Unis, dont le caractère paradoxal et l'ambivalence tiennent des représentations aussi bien positives que négatives de la Chine et des Chinois. La politique extérieure expansionniste des États-Unis, contraste alors avec leur politique intérieure isolationniste: tandis qu'ils prônent une politique de la « Porte ouverte » en Chine, s'engageant à faire respecter le libre commerce dans les zones d'influence occupées par les puissances étrangères, et s'assurant ainsi du libre accès au marché chinois tout en préservant leurs acquis territoriaux, ils optent pour ce que l'auteure appelle une politique intérieure de la "porte fermée ", autrement dit une politique d'immigration hostile envers les Chinois que la société américaine perçoit avant tout comme une menace pour son intégrité raciale et culturelle. La croyance américaine dans la « relation spéciale » qui unit les deux pays et sur laquelle les États-Unis s'appuient pour légitimer les contradictions de leur attitude envers les Chinois, naît donc au XIX ${ }^{\mathrm{e}}$ siècle et préservera toujours le dialogue sino-américain de la rupture irrévocable.

3 La seconde partie met en exergue une autre caractéristique des relations sinoaméricaines, à savoir leur tendance à se laisser infléchir par leurs rapports respectifs avec un pays-tiers. Juliette Bourdin souligne en effet le rôle du Japon dans l'évolution des relations sino-américaines et dans la formulation de la politique chinoise des ÉtatsUnis au cours de la première moitié du XX ${ }^{\mathrm{e}}$ siècle, plus précisément de 1904 - le Japon sort alors vainqueur de la guerre contre la Russie et affirme sa domination sur la scène asiatique sous le regard des grandes puissances occidentales - à 1941, année de l'attaque japonaise à Pearl Harbor qui met fin à la prudence diplomatique observée par les États-Unis et ouvre les hostilités entre les deux pays. Le dialogue sino-américain se complique au tournant $\mathrm{du} \mathrm{XX}^{\mathrm{e}}$ siècle, les Chinois exprimant ouvertement leur indignation face aux traitements réservés à leurs compatriotes en Amérique, à travers le boycott des produits et services américains en 1905. En outre, la prudence américaine face à la puissance nippone favorise le rapprochement de la Chine avec la Russie, dont le modèle bolchévique communiste inspire la création du Parti communiste chinois par Mao Zedong en 1921. La rupture entre le PCC et le Guomindang, prémisse de la guerre civile chinoise, en 1926, compliquera les relations sino-américaines, poussant les États-Unis à la manœuvre diplomatique face aux frères ennemis.

4 Dans la troisième partie, la tendance des relations sino-américaines à s'articuler autour d'un tiers - ennemi et/ou ami - se confirme dans la reconfiguration des alliances militaires imposée par la Seconde Guerre mondiale. L'offensive japonaise sur Pearl Harbor signe la rupture entre le Japon et les États-Unis et favorise un rapprochement sino-américain stratégique, Roosevelt souhaitant inclure la Chine parmi les «quatre grands » pour combattre le Japon. Mais cette alliance, fragile car uniquement stratégique et donc fondée sur des aspirations divergentes, reflète à nouveau la difficulté des deux pays à établir une véritable coopération, s'appuyant sur une entente 
de fond : fin 1943, Roosevelt doit en effet rappeler à Chiang Kai-shek que l'ennemi commun est bien l'envahisseur japonais avant le PCC. Sous la pression du China Lobby, l'administration Roosevelt persiste à soutenir uniquement le régime de Chiang Kaishek, et se laisse déstabiliser par la victoire du PCC en 1948. La place de tiers dans les relations sino-américaines, concédée au Japon pendant l'entre-deux-guerres, se voit désormais occupée par l'Union soviétique, jusqu'alors présente en toile de fond, au début de la Seconde Guerre mondiale: la nouvelle alliance soviéto-américaine est entérinée par les négociations autour de l'entrée en guerre de l'Union soviétique à Yalta en février 1945. Toutefois, la guerre froide, annoncée par la méfiance mutuelle entre le successeur de Roosevelt, Truman, et Staline, va redéfinir les échanges sinoaméricains dès 1947.

5 Le caractère cyclique des relations sino-américaines s'illustre à nouveau dans la quatrième partie, qui s'intéresse aux facteurs de rupture diplomatique entre la Chine et les États-Unis durant la guerre froide, plus précisément de 1949 - lorsque Pékin rejoint officiellement le bloc communiste face à une Amérique en passe de céder au maccarthysme - à 1971, où le successeur de Johnson, Richard Nixon, exprime ouvertement son souhait d'opérer à nouveau un rapprochement avec la Chine. L'auteure souligne l'impact de la guerre froide sur les relations sino-américaines durant les années 1950 et 1960, et par conséquent sur la politique chinoise des États-Unis, dont elle identifie les tendances communes aux administrations Truman, Eisenhower, Kennedy et Johnson. La peur des États-Unis de " perdre la Chine » au profit d'un camp ennemi se trouve ravivée par la guerre froide, s'imposant comme un facteur majeur de reformulation à la fois de la politique intérieure des États-Unis et de leur politique étrangère. Elle motive la mise en place de la politique d'endiguement de Truman ainsi que l'«ambiguïté stratégique»(121) des différentes administrations américaines, soumises à la pression de l'opinion publique et du China Lobby et cependant soucieuses de ne pas déclencher un conflit direct avec la Chine : les États-Unis garantiront en effet leur protection au régime du généralissime à Taiwan tout en s'attelant à freiner les ambitions de "reconquête de la Chine continentale» chez Chiang Kai-shek. La politique chinoise américaine des années 1950 et 1960 se caractérise par son assouplissement et son évolution vers une stratégie d'" endiguement sans isolement " (Containment Without Isolation), formulée par le professeur Arthur Doak Barnett (136) et approuvée par l'opinion publique, pendant les dernières années de la présidence Johnson.

6 La cinquième partie rappelle les intérêts des États-Unis et de la Chine dans la perspective d'un rapprochement et retrace les événements et circonstances qui ont favorisé et compliqué la normalisation des relations sino-américaines de 1972, année $\mathrm{du}$ " voyage pour la paix » (a journey for peace) de Nixon en Chine, à 1989, où la rupture s'opère à nouveau avec les événements de Tian'anmen. L'auteure identifie les caractéristiques de la politique chinoise des administrations Nixon, Ford, Carter et Reagan. Ces dernières convergent vers une normalisation des relations sinoaméricaines et souscrivent à la " diplomatie triangulaire » formulée par Richard Nixon et son Conseiller à la sécurité nationale, Henry Kissinger, et visant à rééquilibrer les rapports de force entre les États-Unis et l'URSS par le biais d'un dialogue sinoaméricain. La nature de cette stratégie diplomatique est ambigüe, officieusement offensive - il s'agit pour les États-Unis de jouer la " carte chinoise " (China card) en exploitant les rivalités et tensions sino-soviétiques pour maîtriser les élans expansionnistes du bloc communiste - et officiellement pacifiste, les États-Unis 
affirmant leur désir de rééquilibrer et d'apaiser leurs rapports de force avec l'URSS. Surtout, c'est une tendance historique des relations sino-américaines qui se vérifie encore et toujours : la construction des dynamiques d'éloignement et de rapprochement entre les deux nations autour d'un pays-tiers, en l'occurrence un rival commun. Toutefois, les différentes administrations américaines, durant les années 1970 et 1980, œuvrent péniblement au rapprochement avec la Chine, toutes faisant face aux divisions générées par la "question de Taiwan", tiraillées par les partisans d'une politique chinoise dure, pro-taiwanaise (celle du Congrès, soutenue par le China Lobby) et les défenseurs d'une ligne de conduite plus souple, conciliante envers la Chine communiste car faisant du rapprochement sino-américain une priorité. Le processus de normalisation des relations sino-américaines, amorcé par Nixon, se poursuit sous Ford et Carter, bénéficiant d'un contexte socio-politique chinois favorable au rapprochement et aux échanges commerciaux sino-américains : Deng Xiaoping impulse en effet un mouvement de modernisation agricole et de libéralisation intellectuelle dans le pays dès 1978. Enfin, l'invasion de l'Afghanistan par le Kremlin en 1979 ravive l'opposition des deux blocs, justifiant davantage le rapprochement stratégique de la Chine et des États-Unis: les relations commencent à s'apaiser à la fin du premier mandat de Reagan et entrent dans l'« ère des bons sentiments " (era of good feeling) à l'issue de la visite du président en Chine en 1984.

7 La sixième partie s'attarde sur la politique chinoise des administrations Bush et Clinton pendant les années 1990. La fin de la guerre froide et la disparition de la menace soviétique redéfinissent les relations sino-américaines, celles-ci ne pouvant plus s'inscrire désormais dans une "relation triangulaire stratégique ": l'" alliance de raison " (167) face à un ennemi potentiel commun n'a plus lieu d'être et c'est finalement l'absence d'un tiers, note Juliette Bourdin, qui va déstabiliser l'équilibre des relations sino-américaines, compliquant le dialogue entre les deux pays durant la dernière décennie $\mathrm{du} \mathrm{XX}^{\mathrm{e}}$ siècle. Ainsi, cette période, marquée par le caractère plus frontal des relations sino-américaines, voit la résurgence et l'accentuation de tendances déjà perceptibles dans les échanges entre les deux pays. La répression des manifestations sur la place Tian'anmen en juin 1989 ravive les dissensions entre le Congrès, qui exige des mesures punitives contre la Chine, et l'administration, menée par l'ancien China Hand George Herbert Bush, qui tient à conserver ses liens d'amitié avec certains dirigeants chinois. Les événements de 1989 introduisent également un nouveau facteur dans l'évolution des relations sino-américaines : si celles-ci hébergent déjà des dissensions idéologiques et des négociations diplomatiques et commerciales, elles sont désormais aussi le terrain d'un affrontement d'ordre moral autour de la question des droits de l'homme. Les considérations économiques constituent le facteur majeur de formulation de la politique étrangère de Clinton, qui se veut l'expression d'un «néo-wilsonisme pragmatique » (pragmatic neo-wilsonism) (183): le modèle économique américain du libéralisme y est envisagé comme le vecteur principal de la diffusion des valeurs américaines et de l'exportation du modèle politique démocratique dans le monde et en particulier en Chine, à laquelle les États-Unis continuent de prêter des aspirations démocratiques. En fait, Clinton s'éloigne d'une politique étrangère fondée sur le principe de containment pour souscrire à une politique d'enlargement et d'engagement avec la Chine. Sensible aux pressions du Congrès, des issue leaders, de plus en plus présents depuis l'émergence de la question des droits de l'homme dans le dialogue sino-américain, et des China Bashers, qui se plaisent à raviver les peurs américaines à l'égard des Chinois - celles de la «menace chinoise» et du "péril 
jaune »- héritées du XIX ${ }^{\mathrm{e}}$ siècle, le président américain tente de placer la question morale des droits de l'homme au centre des relations avec la Chine, articulant l'attribution du statut de la nation la plus favorisée à l'attitude chinoise vis-à-vis du respect des droits de l'homme en 1993. Toutefois, face à l'inflexibilité du PCC et à la montée en puissance économique de la Chine, Clinton est contraint de dissocier la question des droits de l'homme de l'octroi du statut de la nation la plus favorisée à la Chine. C'est avec une approche plus réaliste, plus ancrée dans le contexte économique international, que Clinton est alors contraint d'aborder la politique chinoise pendant son second mandat, se prononçant en faveur de l'entrée de la Chine dans l'OMC. La visite de Jiang Zemin à la Maison-Blanche fin 1997 puis celle de Clinton à Pékin l'année suivante contribuent à une certaine détente des relations entre les deux pays, bien que ces dernières continuent de pâtir d'un certain nombre d'incidents diplomatiques et de la persistance de la peur américaine d'un «complot» de la République populaire de Chine contre l'Amérique.

8 La septième et dernière partie se concentre d'abord sur la politique chinoise de George Walker Bush durant son premier mandat et désigne encore une fois le contexte international, en l'occurrence les attentats du 11 septembre, comme facteur de rapprochement entre la Chine et les États-Unis. La Chine, perçue et traitée comme un "partenaire stratégique» (strategic partner) pendant les années Clinton, est plutôt envisagée comme un "concurrent stratégique» (strategic competitor) par George W. Bush, qui opte pour une politique chinoise plus ferme, visant à éloigner le spectre de l'URSS, dangereusement incarné par la Chine à ses yeux. L'« ambiguïté stratégique » de l'administration Bush se manifeste à travers la politique du « congagement », préconisée par Zalmay Khalizad et soutenue par Condolezza Rice dès la campagne présidentielle de 2000: au croisement des stratégies du containment (adoptée par Truman) et de l'engagement (préconisée par Clinton), cette politique veut se laisser déterminer par l'attitude ouverte ou fermée de la RPC, ainsi que par la montée en puissance de la Chine dans la région Asie-Pacifique. Pourtant, les tensions entre les deux pays sont palpables: Bush ose affirmer publiquement la détermination des États-Unis à défendre Taiwan - ce qu'aucun président n'avait encore fait - attisant la colère de Pékin ; la Chine et la Russie se rapprochent à nouveau; en outre, les États-Unis sont forcés de rappeler à leur partenaire ses engagements commerciaux et les règles de l'OMC. Les attentats du 11 septembre 2001 permettront toutefois aux deux pays d'éviter la rupture définitive - tout comme la guerre du Golfe a finalement poussé la Chine et les États-Unis au rapprochement pendant la présidence de George Herbert Bush - les obligeant à dépasser les tensions pour coopérer dans la "guerre contre le terrorisme " (War on Terrorism). Le rôle de la Chine dans la lutte de Bush contre l'« axe du mal » s'avère en effet fondamental, notamment dans les négociations sur la dénucléarisation de la Corée $\mathrm{du}$ Nord, dont elle est le " grand frère communiste ", ainsi que dans les discussions au Conseil de Sécurité de l'ONU autour d'une intervention américaine en Irak, intervention possible seulement si la Chine s'abstient de voter contre une opération qu'elle ne soutient pas. Réciproquement, la Chine a tout intérêt à ce que ses relations avec les États-Unis s'apaisent si elle veut préserver son équilibre économique et continuer à se moderniser. Les relations sino-américaines ont donc «bénéficié d'une embellie conjoncturelle " (209), même si une méfiance mutuelle demeure. Juliette Bourdin termine cette dernière partie en adoptant une lecture thématique de la dynamique et des enjeux actuels et futurs des relations sino-américaines, ainsi que de leur impact dans le monde et la région Asie-Pacifique. Elle souligne la persistance, au 
$\mathrm{XXI}^{\mathrm{e}}$ siècle, du caractère pendulaire des relations entre les deux pays, engagés tantôt dans un "duo ", tantôt dans un "duel " et s'interroge sur la pertinence actuelle et l'avenir de ce que Niall Ferguson et Morick Schularick nomment "Chimerica», soit la "Chinamérique », désignant l'interdépendance économique et financière des deux pays tout en suggérant sa nature éphémère, «chimérique » (211-212) : si les États-Unis, en déficit commercial avec la Chine, dépendent de celle qui est devenue leur principal créancier depuis 2008, la Chine dépend des États-Unis et de leur intérêt pour son "marché du milliard», mythe qu'elle doit entretenir pour continuer son ascension économique. Cette interdépendance, reconnue par exemple à travers l'US-China Strategic and Economic Dialogue, institué en avril 2009 par Barack Obama et Hu Jintao, impose de part et d'autre une stratégie de "dissuasion économique» (220) assurant l'équilibre économique entre les deux pays, ce qui explique la politique plus modérée d'Obama, qui à l'instar de son concurrent et contrairement à ses prédécesseurs, s'est gardé d'utiliser la Chine comme thème de campagne électorale pour critiquer l'administration sortante en 2008 et s'est montré favorable à une politique clintonienne d'engagement; en atteste sa réticence à évoquer la question des droits de l'homme lors de son premier voyage en Chine. Enfin, l'auteure revient sur les principaux enjeux stratégiques toujours à l'œuvre dans les relations sino-américaines. La "question de Taiwan» demeure épineuse et entretient les tensions entre Pékin, Taipei et Washington: face au nationalisme affiché de Taiwan, en particulier jusqu'en 2008, la Chine s'est montrée ferme avec le vote de la loi anti-sécession le 14 mars 2005 et n'écarte pas le recours à la force pour récupérer l'île, tandis que les États-Unis souhaitent une résolution pacifique de la question. Toutefois, elle impose une prudence mutuelle entre la Chine et Taiwan, qui optent, avec la médiation des États-Unis, pour l' "ambiguïté stratégique ». En outre, soucieuse de recouvrer sa grandeur passée et d'assurer son influence en Asie, la Chine s'adonne à une "pétrodiplomatie active " (229) auprès de ses voisins en Asie-Pacifique : dans cette perspective, les relations sinorusses jouissent d'une embellie depuis le début du siècle ; les relations sino-japonaises, en revanche, sont toujours alourdies par le traumatisme de l'invasion japonaise pendant la Seconde Guerre mondiale, ainsi que par la rivalité historique entre les deux pays pour une domination économique et militaire en Asie, où là encore le soutien américain joue un rôle déterminant. Alors que les intérêts économiques et géopolitiques de la "Chinamérique » laissent penser que les deux pays abordent le $\mathrm{XXI}^{\mathrm{e}}$ siècle en tant que « duo ", la croyance dans la « menace chinoise » est toujours présente aux États-Unis, la perspective éventuelle d'une superpuissance chinoise qui détrônerait les Américains sur la scène internationale inquiétant le China Lobby. Mais pour Juliette Bourdin, de telles craintes sont suscitées davantage par le «potentiel» chinois, plus que par une réelle supériorité économique et militaire de la Chine sur les États-Unis : «à bien des égards, la perception de la puissance chinoise est supérieure à sa puissance.» (Daguzan, 2004, 17) Les représentations américaines de la Chine sont donc vivaces : si les États-Unis diabolisent encore la Chine en raison de son potentiel et de ses ambitions, ils continuent de nourrir également le rêve d'une Chine en passe de se démocratiser (/s'américaniser) et de confirmer ainsi l'« universalité » du modèle sociopolitique et moral occidental (235). 


\section{Critique de l'ouvrage}

9 Entre porte ouverte et " porte fermée » constitue un apport précieux à l'historiographie des relations sino-américaines, notamment parce qu'il s'adresse à un public francophone : si les synthèses historiques retraçant l'évolution des rapports entre la chine et l'Amérique sont en effet nombreuses aux États-Unis - l'auteure mentionne celle de Michael Schaller, The United States and China (2002) et celle de Warren I. Cohen, America's Response to China (2010) comme les études de référence dans le domaine anglophone l'analyse de Juliette Bourdin s'impose comme la pionnière dans la littérature francophone sur l'évolution et les enjeux des relations sino-américaines.

10 L'objectif de l'étude est ambitieux et novateur puisqu'il s'agit pour l'auteure d'opérer un retour chronologique sur les deux siècles de l'histoire des relations sino-américaines et ainsi de permettre aux lecteurs d'apprécier les tensions et les enjeux qui les caractérisent aujourd'hui, leurs "réalités actuelles » à la lumière aussi bien des conjonctures passées que de la facture socio-culturelle de chaque partie telle qu'elle s'est révélée et affirmée au gré des échanges, influant sur la dynamique relationnelle sino-américaine, entre rapprochement et éloignement. L'esquisse de ce parcours des relations entre la Chine et l'Amérique distingue la synthèse de Juliette Bourdin des autres études, la plupart optant pour une analyse synchronique du dialogue sinoaméricain privée d'un regard rétrospectif suffisamment averti sur les origines et les remous des relations entre les deux pays (20).

11 La structure de l'ouvrage témoigne du souci pédagogique de Juliette Bourdin de guider son lecteur: les temps de synthèse proposés par l'auteure au début et à la fin de chacune des sept parties, ainsi que dans la conclusion de son analyse, s'avèrent précieux pour le lecteur confronté à la densité et à l'abondance des événements ayant marqué les relations sino-américaines pendant près de deux siècles. Ils sont l'occasion pour Juliette Bourdin de rappeler de façon succincte les tendances dont elle vient minutieusement de contextualiser l'émergence et la résurgence, et ainsi d'offrir au lecteur une esquisse indispensable de la dynamique relationnelle entre les deux pays durant la période analysée.

12 La perspective diachronique adoptée par Juliette Bourdin est efficacement servie par le choix de structuration de l'ouvrage: de la lecture chronologique de l'histoire des relations sino-américaines à la réflexion thématique autour des enjeux économiques et stratégiques actuels inhérents aux rapports entre les deux pays et par conséquent, des possibles prolongements et développements du paradigme relationnel sino-américain. Si l'approche chronologique, privilégiée tout au long de l'ouvrage, peut faire craindre la redondance dès lors qu'elle invite l'auteure à identifier et à signaler les tendances caractéristiques des relations sino-américaines à chacune de leur résurgence au cours des deux derniers siècles, elle permet justement de souligner le rôle fondamental des différentes conjonctures nationales - proprement américaine et chinoise- et internationales traversées par ces relations dans l'émergence et la répétition cyclique de ces tendances. Une approche essentiellement thématique, s'articulant d'emblée autour des tendances relationnelles préalablement identifiées, définies et de ce fait, impliquant une évocation plus parcellaire, car orientée, ciblée, des différents contextes historiques et socio-politiques ayant contribué à l'élaboration de la dynamique sinoaméricaine, risquerait de passer sous silence les facteurs invisibles - irréductibles aux événements, aux circonstances et aux faits - de l'évolution du dialogue entre la Chine 
et les États-Unis. En retraçant la genèse et le parcours compliqués des relations sinoaméricaines, Juliette Bourdin nous fait prendre la mesure de la dimension humaine de ce dialogue et de ce lien entre deux nations, deux cultures, deux imaginaires collectifs se rejoignant et se heurtant au gré des aléas de l'histoire mais aussi des malentendus, des impairs et des secrets diplomatiques, suscités par les espoirs, les projections et les peurs de chaque partie, qui viennent percer l'évocation linéaire des événements.

13 L'analyse minutieuse et rigoureuse de Juliette Bourdin nous semble mettre en lumière principalement deux grands paradoxes caractéristiques des relations entre les deux pays. Il apparait d'abord que les échanges sino-américains, en dépit des dissensions idéologiques, des conflits d'intérêts et des quiproquos diplomatiques qui les ont marqués, confirment les traits distinctifs de la politique américaine étrangère en général. La Chine, interlocuteur historique des États-Unis à la fois retors, souvent rétif et néanmoins toujours nécessaire, "semble agir comme un puissant révélateur des facteurs qui pèsent en général sur la politique étrangère américaine " (242). Les différences structurelles entre les deux pays, et par conséquent le caractère versatile de leurs rapports, poussent les États-Unis à affirmer l'identité américaine de leur politique extérieure.

Les deux principaux facteurs de (re)formulation de la politique chinoise des États-Unis se trouvent en effet clairement illustrés au fil de l'ouvrage: "l'immixtion de la politique intérieure dans la politique chinoise de Washington " s'impose comme une « constante » (242) dans les relations sino-américaines, qui se sont vues régulièrement influencées par des problématiques et des enjeux propres au contexte socio-politique américain. L'étude de Juliette Bourdin insiste en effet sur les tensions entre le Congrès, s'appuyant sur le discours farouchement anti-communiste et pro-taiwanais du China Lobby surtout jusque dans les années 1990, et l'administration, toujours soucieuse de ne pas provoquer la rupture définitive avec l'interlocuteur chinois. Le double impact de telles divisions sur les relations sino-américaines est souligné au fil de l'ouvrage. D'une part, l'ambivalence de la politique chinoise de Washington est illustrée notamment par le recours des administrations Nixon et Bush dans les années 1970 et 1980 à la «diplomatie du secret » pour préserver l'entente avec les dirigeants chinois tout en répondant aux attentes du Congrès; d'autre part, le manque de lucidité et de pragmatisme de la politique chinoise américaine, grevée des attentes idéologiques et morales du China Lobby, est manifeste dans le déni de l'administration Roosevelt face à la montée en puissance du PCC pendant la guerre civile chinoise - «La stratégie américaine sera victime de son manque de connaissance et de compréhension de la situation chinoise, puis du regard biaisé porté sur les communistes chinois » (80), explique Juliette Bourdin - ainsi que dans la politique utopique de Clinton, dont le tort aura été de faire de la question morale du respect des droits de l'homme une priorité dans une période d'interdépendance économique croissante entre la Chine et les ÉtatsUnis. Un contexte économique qui a vite contraint le président d'«abandon[ner] le "néo-wilsonisme" pour ne conserver que le "pragmatisme", autrement dit [de passer] de l'idéalisme au réalisme. » (186) De façon intéressante, l'auteure fait remarquer la tendance inverse qui infléchit aussi les relations sino-américaines, à savoir l'exploitation de la Chine par les acteurs de la politique intérieure américaines: la paranoïa qui agite l'Amérique maccarthyste des années 1950 et 1960 est, selon l'auteure, « l'expression la plus caricaturale de l'utilisation de la Chine dans la politique intérieure des États-Unis " (118); la Chine s'avère aussi être un thème électoral utile, largement mis à profit par les présidents Clinton et Bush - le premier en 1992, le 
second en 2000 - qui s'emploient à souligner les failles de l'administration sortante et à critiquer son attitude (jugée toujours trop conciliante par le Congrès) face à l'interlocuteur chinois.

15 Si cette constante conjoncturelle des relations explique en partie le caractère ambivalent, "bicéphale " de la politique chinoise de Washington, un facteur plus structurel, intrinsèque à l'identité nationale américaine, permet aussi d'en comprendre la versatilité, à savoir "l'idéologie et l'image que les États-Unis ont d'eux-mêmes » (242) et qui nécessairement déterminent la représentation américaine de la Chine. Ainsi, l'auteure démontre de façon tout à fait convaincante que les complications du dialogue sino-américain sont nées principalement du décalage entre la réalité et les « images manichéennes» que les États-Unis entretiennent de la Chine, celles d'une "good China", d'une Chine aspirant à embrasser le modèle démocratique occidental (américain), et d'une "bad China» (241), d'une Chine hostile car obstinément communiste. Elle note ainsi «la distorsion de la vision américaine de la Chine qui, fatalement, influe sur le cours des relations sino-américaines: la grille d'analyse, brouillée auparavant par le mythe paternaliste de la "relation spéciale", [s'est ensuite vue] déformée par celui de la "perte de la Chine".» (120) L'ouvrage montre clairement la persistance de ces deux perceptions américaines des relations avec la Chine, la première ayant engendré la seconde, insiste l'auteure. Le mythe de la «relation spéciale ", né au XIX ${ }^{e}$ siècle des ambitions expansionnistes et civilisatrices des ÉtatsUnis, qui continuent à se penser en modèle idéologique, social, moral et politique pour la Chine, permet aux Américains de légitimer l'ambivalence de leur politique chinoise, tout comme elle sert les fins stratégiques des dirigeants chinois : ainsi, pendant l'entredeux-guerres, Chiang Kai-shek, n'hésite pas à invoquer la « relation spéciale » tout en en suggérant la fragilité et parvient de cette façon à faire reconnaître le parti nationaliste par les États-Unis, à l'aube de la guerre civile chinoise. Le mythe de la "perte de la Chine» résulte de la croyance des États-Unis dans leur «relation spéciale " avec la Chine, autrement dit dans le développement parallèle des deux nations, culturellement opposées et cependant vouées à se rejoindre dans une destinée - démocratique et libérale- commune. Aussi le parcours socio-politique et économique chinois suggère-t-il un dénouement inenvisageable pour les États-Unis " [c]ar si les deux pays sont censés être tellement similaires, l'Amérique ne court-elle pas autant de risques de subir le même sort que celui de la Chine, à savoir de tomber sous le joug communiste?» (118) Les années maccarthystes frappent ainsi par leur caractère conjuratoire, tout comme elles révèlent la certitude des États-Unis selon laquelle le régime communiste ne peut être que transitoire en Chine et est voué à l'échec. Bien que cette conviction ait été ébranlée par la survivance du PCC en dépit du schisme sino-soviétique de 1960 puis du déclin de l'URSS et de la fin de la guerre froide en 1989, les États-Unis continuent à nier la solidité du modèle socio-économique et idéologique chinois, qui contredit « la philosophie politique américaine », celle-ci « ne [pouvant] concevoir que le développement du capitalisme économique puisse aller de pair avec le maintien d'un régime autoritaire, a fortiori communiste." (133) Ainsi, l'auteure suggère que c'est l'identité américaine qui se joue dans le déni des États-Unis face à la résilience du communisme chinois. Juliette Bourdin fait remarquer la pertinence et la résonance cycliques de la thèse de la " perte de la Chine » au cours de l'histoire des relations sino-américaines : elle est à l'origine de l'engagement américain dans la guerre de Corée de 1950 à 1953 puis dans la guerre du Vietnam de 1955 à 1975, face à une Chine militairement présente dans les deux pays et affirmant son 
appartenance au bloc communiste pendant la guerre froide. C'est également cette peur de la "perte de la Chine » qui explique les contorsions diplomatiques des États-Unis face à la « question de Taiwan », les administrations américaines affirmant leur soutien et leur protection au régime non communiste de Taiwan - en particulier par leur refus de cautionner la réunification de lîle à la Chine continentale par la force - tout en reconnaissant le régime communiste au pouvoir en Chine afin de ne pas rompre le dialogue : à l'exception de George W. Bush, les présidents américains, dont Barack Obama, se sont tous prononcés en faveur de la "politique d'une seule Chine " (OneChina Policy), officiellement reconnue dans le Communiqué de Shanghai en 1972. Mais l'un des intérêts indéniables de l'analyse de Juliette Bourdin réside surtout dans sa réflexion autour des implications identitaires du mythe de la " perte de la Chine » qui se trouve étroitement lié à la « perception que l'Amérique a d'elle-même » (Isaacs, 1958, 191) : « perdre la Chine » pour les Américains signifie être confronté aux limites de leur modèle socio-politique et de leurs valeurs. Tantôt «idéalisée ", tantôt "diabolisée » (241) dans la perception américaine, la Chine a donc toujours échappé aux États-Unis qui ont davantage dialogué avec leur représentation de la Chine qu'avec la Chine ellemême. " [P]arce que les relations entre les deux pays ne reposent finalement sur aucune base solide », (242) la politique chinoise de Washington a souvent manqué de lucidité et de réalisme, se laissant infléchir par les acteurs de la scène politique intérieure américaine et nourrissant le rêve utopique d'une Chine à l'image de l'Amérique. Le président Clinton, contraint de renoncer à lier le statut de la nation la plus favorisée à la question des droits de l'homme face à l'inflexibilité des dirigeants chinois, a ainsi appris que le cheminement de la Chine vers la démocratie ne se ferait pas sous la pression étrangère et que négliger la réalité de la culture, de l'histoire et du développement socio-politique propres à la Chine mènerait irrémédiablement le dialogue entre les deux pays dans l'impasse.

L'autre paradoxe des relations sino-américaines, mis en exergue par la lecture de Juliette Bourdin, résulte précisément des visions antinomiques de la Chine au cœur de la politique chinoise des États-Unis : parce qu'elles reposent principalement sur les projections de l'une et de l'autre partie, et par conséquent sur une base fragile, les relations entre les deux pays semblent ne pouvoir trouver un apaisement et une certaine stabilité que face à l'adversité commune, lorsque le rapprochement, voire l'alliance, stratégique est nécessaire. C'est dans la coopération, plus que dans l'entente de fond, que résident la force et la résilience des relations sino-américaines. L'auteure fait d'ailleurs remarquer que la politique chinoise de Clinton a pâti d'un contexte international apaisé dans les années 1990, soit de l'absence d'une menace commune nécessitant l'alliance des deux interlocuteurs et surtout le rassemblement des différents acteurs politiques américains autour d'une ligne de conduite commune, cohérente, vis-à-vis de la Chine : « depuis la Seconde Guerre mondiale, Bill Clinton a été le seul président à bénéficier d'un contexte international aussi peu troublé, mais il a subi, dans une certaine mesure, l'“absence" d'un ennemi qui lui aurait permis de transcender les problèmes internes pour rassembler les citoyens autour d'une cause commune et s'en remettre à l'action du président. » (198)

17 L'analyse de Juliette Bourdin fournit les clés nécessaires à la lecture des enjeux actuellement à l'œuvre dans le dialogue entre Obama et Xi Jinping, où l'on constate la résurgence des tendances historiques des relations sino-américaines. L'«ambiguïté stratégique » est toujours de mise dans la politique chinoise d'Obama, qui se définit comme « un compromis entre des éléments du hard power (militaire, économie) et du 
soft power (influence, dialogue, persuasion) » (Courmont, 2012, 93) : autrement dit, en affirmant la vitalité et la compétitivité économiques ainsi que la puissance militaire des États-Unis tout en privilégiant la discussion et la négociation, Obama entend préserver l'équilibre des pouvoirs et des influences dans les relations sino-américaines. Si la "diplomatie intelligente» (smart power) et le recours au dialogue sont les voies privilégiées par Obama, les relations entre les deux pays n'en demeurent pas moins ambivalentes. Conscients de la nécessité d'une coopération sino-américaine solide, qu'Obama souligne lors de la visite du président chinois à Washington en février 2014, la Chine et les États-Unis continuent cependant de s'affronter pour une domination économique notamment en Amérique latine - dont les besoins d'emplois manufacturiers suscitent l'intérêt des entreprises chinoises et par conséquent des entreprises américaines - et en Asie-Pacifique: Washington et Pékin se dispute la région par le biais du Trans-Pacific Partnership (TPP), dont la Chine et la Russie sont exclues, et le Free Trade Area of the Asia Pacific (FTAAP), proposé par les Chinois pour concurrencer le TPP. En outre, malgré sa réticence à évoquer les questions d'ordre moral avec son homologue chinois au début de son mandat, Obama n'a pas hésité à rappeler l'importance du respect des droits de l'homme et de la liberté de la presse pour les États-Unis face à Xi Jinping, en février dernier, révélant ainsi l'espoir vivace des États-Unis de voir la Chine adopter bientôt leur modèle démocratique.

Notons enfin que cet ouvrage présente également un intérêt certain pour les études littéraires sino-américaines dans la mesure où il apporte un éclairage international sur le rapport de la société américaine à ses communautés asiatiques et en particulier à sa communauté chinoise, depuis l'arrivée des premiers immigrants aux États-Unis à la fin du XIX ${ }^{e}$ siècle. De cette façon, elle rend possible la relecture des conditions sociopolitiques d'émergence et du parcours de cette communauté - dont une grande partie de la production littéraire est autobiographique - dans la société américaine, à la lumière des relations diplomatiques, des tensions et des enjeux idéologiques, politiques, économiques et militaires entre le pays d'origine et le pays d'accueil de ses immigrants. L'ambivalence de la société américaine envers sa communauté chinoise, sentiment autour duquel s'articulent les enjeux identitaires au cœur de la production des écrivains américains d'origine chinoise, trouve ainsi une résonnance plus large dans cette analyse de la "relation d'amour/haine» (love/hate relationship) (McGiffert, 2005, xiii) qui unit et sépare la Chine et les États-Unis.

INDEX

Thèmes : Recensions

\section{AUTEURS}

NELLY MOK

Université Paul Valéry Montpellier 